\title{
The circadian clock of cyanobacteria
}

\author{
Takao Kondo* and Masahiro Ishiura
}

\begin{abstract}
Summary
A circadian clock, with physiological characteristics similar to those of eukaryotes, functions in the photosynthetic prokaryote, cyanobacteria. The molecular mechanism of this clock has been efficiently dissected using a luciferase reporter gene that reports the status of the clock. A circadian clock gene cluster, kaiABC, has been cloned via rhythm mutants of cyanobacterium, Synechococcus, and many clock mutations mapped to the three kai genes. Although kai genes do not share any homology with clock genes so far identified in eukaryotes, analysis of their expression suggests that a negative feedback control of kaiC expression by KaiC generates the circadian oscillation and that KaiA functions as a positive factor to sustain this oscillation. BioEssays 22:10-15, 2000.

(c) 2000 John Wiley \& Sons, Inc.
\end{abstract}

\section{Introduction}

Circadian rhythms, oscillations with approximately $24 \mathrm{~h}$ periods in many physiological activities, are found in a wide spectrum of organisms. ${ }^{(1)}$ Three distinct characteristics of circadian oscillation are required in order that these rhythms function as a "clock." These are: (1) persistence of the rhythms in the absence of external time cues; (2) resetting the phase by light or dark; and (3) temperature compensation of the circadian period. Thus, the circadian clock that controls the rhythm is assumed to be a fundamental function of living cells to adapt to the alternation between day and night.(2) Physiological studies have elucidated the basic structure of many circadian systems; it is composed of a central oscillator located within the cell which controls output pathways that drive the observable rhythms. The phase of the oscillation is set by an input pathway that responds to external time cues. The molecular mechanisms that generate the oscillation remained a mystery for many years, however. Attempts to study the clock by pharmacological or biochemical approaches were impeded by the fact that the

Division of Biological Science, Graduate School of Science, Nagoya University, Chikusa, Nagoya, Japan.

Funding agencies: Japanese Ministry of Education, Science and Culture; Grant numbers: 11233203, 11440234, 1151213, 11558089; Research for the Future Program of the Japan Society for the Promotion of Science; Grant number: JSPS-RFTF96L00601; Human Frontier Science Program.

*Correspondence to: Dr. Takao Kondo, Division of Biological Science, Graduate School of Science, Nagoya University, Furo-cho, Chikusaku, Nagoya 464-8602, Japan. E-mail: kondo@bio.nagoya-u.ac.jp rhythms measured in these studies were merely the "hands" of the clock.(3) More recently, molecular genetic approaches in Drosophila, Neurospora, and mice have revealed the basic mechanism of the oscillator at the molecular level. $(4,5)$

\section{Finding the circadian clock in cyanobacteria}

Following several negative results in identifying time-dependent fluctuations in bacteria, the circadian clock was initially assumed to exist only in eukaryotes. However, in the last decade, circadian rhythms have been reported in several strains of nitrogen-fixing cyanobacteria (reviewed in Ref. 6) Alternation of day and night dominates the metabolism of photoautotrophic cyanobacteria that fix nitrogen. During daylight, photosynthesis by these bacteria generates oxygen from water, but since the nitrogenase enzyme is sensitive to oxygen, nitrogen fixation in cyanobacteria has to be isolated from this oxygen. Filamentous cyanobacteria solve this problem by the development of specialized cells called heterocysts. In these multicellular species, vegetative cells produce oxygen through full-chain photosynthesis but do not fix nitrogen, while heterocysts turn off photosystem II when they express the nitrogenase enzyme. This spatial segregation is clearly not possible in unicellular species, however. In these, the temporal separation of the two incompatible activities - photosynthesis in the day and nitrogen fixation during night - has been observed. Mitsui et al. ${ }^{(7)}$ were the first to describe a daily oscillation of nitrogenase activity of a marine cyanobacterium. Huang and coworkers later reported the circadian rhythms of nitrogenase activity and amino acid uptake in a freshwater nitrogenfixing strain, Synechococcus sp. RF1. They demonstrated that period of these rhythms were temperature compensated, and the phases were reset by light signals. ${ }^{(8-10)} A$ circadian rhythm in cyanobacterial cell division was also reported by Sweeney et al.(11) and the rhythms of photosynthesis and/or nitrogen fixation observed in the cyanobacteria, Cyanothece(12) and Trichodesmium.(13) These findings suggested two points: (1) circadian oscillators can function in the absence of eukaryotic cell structure; and (2) cyanobacteria could be a model system for molecular approaches to the circadian clock, because it is the simplest organism known to possess this activity.

\section{An artificial "hand" of the clock - a luciferase reporter gene}

Bioluminescence provides a continuant method to monitor circadian rhythm, as exemplified by many studies with the 
bioluminescent dinoflagellate, Gonyaulax.(14) The light output can be measured with great sensitivity without destroying the cells. Therefore, designing a bioluminescence system that reports a status of the oscillator could greatly facilitate rhythm analyses. If the activity of a particular gene promoter were controlled by an endogenous circadian clock, luciferase DNA inserted downstream of such a promoter should be expressed under clock control. By continuously administering the luciferase substrate, the rhythmic control of the circadian clock over the target promoter should be monitored as daily oscillations in the intensity of bioluminescence. This strategy has been successfully applied also in Arabidopsis(15) and Drosophila. (16)

We selected a strain of cyanobacterium, Synechococcus sp. PCC 7942 (Synechococcus hereafter), that is easily transformed by exogenous DNA and is widely used in molecular genetic studies.(17) The size of this organism's genome is $2.7 \mathrm{Mb}$, which is smaller than that of E. coli. Synechococcus cells were transformed with a construct containing the promoter of the $p s b A$ gene that encodes a major component of the photosystem II reaction center (D1 protein(18)) driving expression of the bacterial luciferase (luxAB) gene (Fig. 1). LuxAB was chosen because its volatile, hydrophobic substrate, $n$-decanal, diffuses directly into Synechococcus. Bioluminescence of the transformed Synechococcus cells oscillate with a period of approximately $24 \mathrm{~h}$ for more than 10 cycles in constant light conditions (LL). Moreover, the phase of the rhythm is reset by a single dark pulse in a phase-dependent manner and the period length is little altered by temperature. Therefore, the rhythms of prokaryotic Synechococcus satisfy the three salient criteria for circadian rhythms that are established in eukaryotes.(19) This transformed Synechococcus is one of the simplest model systems for the circadian oscillator.

\section{Temporal orchestration of gene expression in cyanobacteria}

Initially, the $p s b A$ I gene was considered unique in being under a control of the circadian clock; hence, it seemed highly fortunate that this promoter was chosen for these experiments. A promoter trap strategy revealed that the circadian clock in Synechococcus dominates the entire metabolism of the organism, and almost every gene is controlled by the circadian clock. (20) In this comprehensive search for genes expressed under clock control, Synechococcus genomic DNA fragments were fused to the promoter-less I $\mathrm{x} A B$ gene and the fusion transformed into wild-type Synechococcus. By homologous recombination into the chromosome, the region of DNA surrounding the insertion site was expected to be duplicated so that the intact original gene and the reporter fusion should remain after the insertion event. By monitoring bioluminescence of transformants, therefore, it is possible to assay promoters that are included in the library insert DNA ("promoter

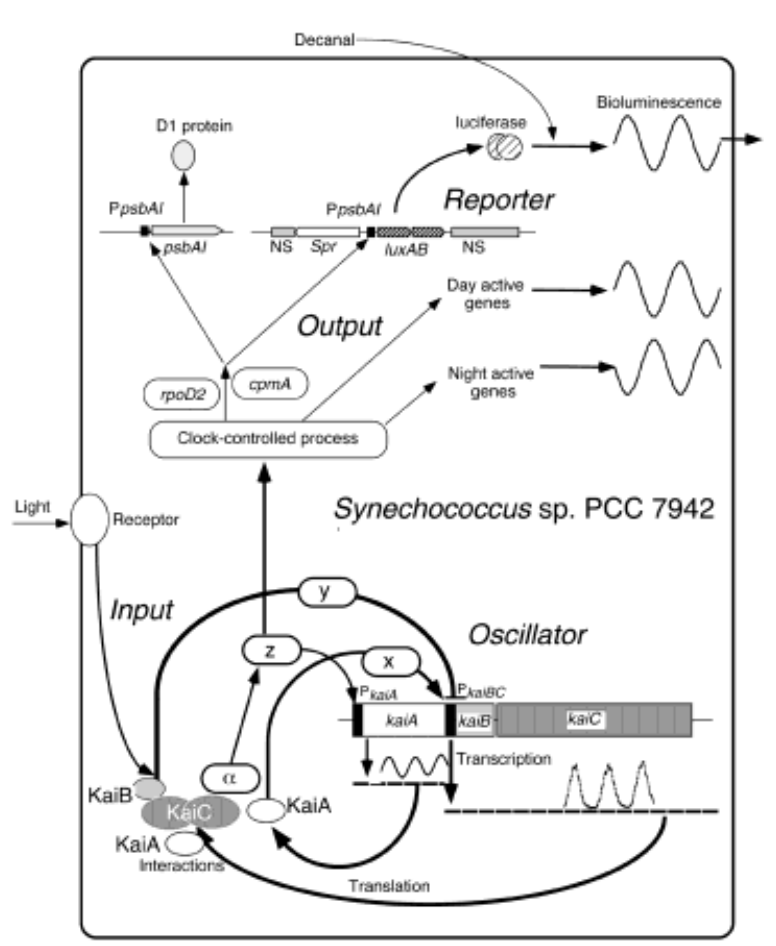

Figure 1. Circadian organization of Synechococcus. This organization is composed from an Oscillator, an Input and an Output, as illustrated. Oscillator: In this prokaryotic model, kaiC expression is regulated both by positive action through KaiA and by negative feedback by KaiC. $\alpha, \mathrm{x}, \mathrm{y}$ and $\mathrm{z}$ represent possible transcription factors, as yet unidentified. Input: External light signals are transferred to the oscillator to reset the phase of the oscillation; the relevant receptor molecule(s) and their action on the oscillator are unknown. Output: Through unknown transcription factor(s), the oscillator controls a diverse set of gene expression. A pathway that is influenced by rpoD2 and $c p m A$ regulates $p s b A l$ and other genes. Reporter: The PpsbAl:IuxAB construct is inserted in to a NS (neutral site in the genome $\left.{ }^{(19)}\right)$ and, experimentally, serves as an artificial hand of the clock. By administrating the luciferase substrate (decanal), the reporter strain bioluminesces with a circadian rhythm, which is altered, often dramatically, by induced mutations, most of which are in the kai gene cluster.

trap"). Unexpectedly, all of the bioluminescent transformants (800 clones) of Synechococcus expressed clear rhythmic patterns.

The activity of up to $80 \%$ of the promoters assayed oscillate with a phase similar to that of PpsbAl in which bioluminescence peaks near the end of day. Some of these genes are likely to be directly regulated by the clock via cis elements and trans factors, which coordinately regulate the expression of groups of genes. It seems unlikely that each of them is controlled by a specific regulatory factor, however. Some global factor(s) that influence the transcriptional rate 
of many genes are also thought to be involved. For example, the rpoD2 gene that encodes a sigma factor of RNA polymerase $^{(21)}$ and recently identified cpmA gene(22) control a subset of these genes. Moreover, rhythmic alteration in energy metabolism, such as redox state or energy charge, could influence the transcriptional rate of many genes. Some clones oscillate in the reverse phase to that of the PpsbAl promoter, however. Possibly, this expression pattern would apply to genes encoding enzymes that are oxygen sensitive; because photosynthesis inevitably generates oxygen, the activation of these enzymes might be best performed primarily in the night. purF, which encodes an oxygen-sensitive enzyme of the purine nucleotide biosynthetic pathway, ${ }^{(23)}$ is an example of such a gene.

\section{Adaptive significance of circadian clock in cyanobacteria}

It is widely believed from an evolutionary perspective that circadian clocks improve survival by facilitating adaptation to the environment.(2) More specifically, circadian regulation enables a predictive control to achieve the maximum efficiency in metabolism, whereas passive response to environment signals would be reactive. In unicellular cyanobacteria, the circadian clock could precisely regulate photosynthetic genes to be active during day and genes involved in nitrogen fixation to be active at night. However, the merit of circadian control is not always so obvious. In many species, clock mutants (including arrhythmic mutants) survive as well as wild-type organisms, at least in laboratory conditions. This casts doubt upon the adaptive significance of the circadian clock. Enhanced fitness of wild-type cyanobacteria has been demonstrated by competition experiments, however.(24) In these studies, wild-type and period mutants were cultured in the same test tube, so that the two strains competed against each other for their growth under exactly the same conditions. The results clearly indicated that the strain with the period most similar to that of the external light/dark cycles was most successful. Similar results have been obtained in the studies of the lifespan of Drosophila clock mutants. (25)

\section{Synechococcus as a model system for molecular genetic dissection of the circadian oscillator}

Bacterial molecular genetics has been facilitated by the ability to detect mutant phenotypes of individual colonies on agar plates. Using a cooled-CCD camera and a customdesigned software, it is possible to monitor the circadian rhythms of more than 10,000 independent colonies simultaneously. From 500,000 clones that were mutated with ethylmethanesulfonate, more than 100 clock mutants were isolated.(26) Period mutants exhibited robust rhythms with altered periods of between 14 and $60 \mathrm{~h}$, and other mutants showed arrhythmia and distorted waveforms. All rhythmic strains were entrained by dark pulses and were able to compensate for temperature. These mutations appear clock-specific because all the mutant strains grow as well as wild-type cells.(26)

\section{The kai gene cluster as a central component of the cyanobacterial clock}

Efficient genetic complementation of mutant phenotypes is possible in Synechococcus by introduction of a wild-type genomic DNA library into an array of cells. In this way, a gene cluster composed of three novel open reading frames was identified in DNA fragments recovered from rescued clones of several different rhythm mutants.(27) Following this, more than 25 mutations - including short-period, longperiod, low amplitude, and arrhythmic phenotypes - were mapped to these three ORFs. This gene cluster was named kai (cycle in Japanese), with the ORFs termed kaiA, kaiB, and kaiC (Fig. 1). (27)

Interestingly, amino acid sequences of Kai proteins do not share homology to any clock genes so far identified in other organisms. ${ }^{(5)}$ Deletion of the kai gene cluster or inactivations of each kai gene individually completely abolished rhythmicity, and re-introduction of the whole kai gene cluster restored the rhythm. (27) From this, it is evident that all three kai genes are crucial for the circadian rhythm of Synechococcus. Moreover, more than 50 clock mutants so far examined were all complemented by introduction of DNA from the kai gene cluster, regardless of the diverse mutant phenotypes. These results imply that the function of kai genes represent the principal feature of the cyanobacterial circadian clock. In addition, the normal growth of a kai knockout clone suggests that kai genes are dedicated to the generation of circadian oscillations. It is possible that other genes that are also important for clock function might contribute to cellular metabolism in other ways and so be discriminated against the screening strategy that requires normal growth. At present, a pex gene, mutation of which extends the circadian period by $1 \mathrm{~h}$, is the only rhythm-related gene found in Synechococcus. ${ }^{(28)}$

\section{Molecular cycling models for the circadian oscillator}

In the absence of external time cues, circadian rhythms persist for many cycles, sometimes for a half year. Thus, the circadian oscillator is a self-sustaining oscillator, and damping observed in constant conditions could be attributed to difficulties in culturing organisms under the conditions that allowed assay of such oscillations. Self-sustained oscillations are found everywhere in nature, from physical oscillations to ecological phenomena and can be discussed by using a mechanical analogy. Assume an object is connected to a wall by a spring and placed on a conveyer belt that moves in one direction at constant speed. If friction between

\section{BioEssays 22.1}


the object and the belt is not influenced by the relative velocity of the object, forces associated with friction and the spring will be balanced, and the oscillation of the object will damp quickly. In reality, however, friction is slightly larger when the relative speed is low. So, moving the object by friction will lower the relative speed and further increase the friction. This positive feedback will make the deviation of the object larger, while the force of the spring always acts to restore the deviation of the object (negative feedback). As a result of positive and negative feedback actions upon the object, the object will generate the stable oscillation.

In biology, similar mechanisms could operate in many ultradian rhythms (whose periods are shorter than $24 \mathrm{~h}$ ), such as those seen in cytoplasmic streaming of Physarum or glycolysis of yeast extracts. ${ }^{(29)}$ In the circadian oscillator, negative feedback associated with the transcription and translation of specific gene(s) could be involved. In this regard, clock genes cloned in Drosophila (per and tim), Neurospora (frq), and Synechococcus (kaiC) could function as key components to define the oscillation, as the object in the physical models does, because these genes satisfy the following fundamentals: (1) Mutations of these genes significantly alters the circadian phenotype; (2) null mutations abolish more than one kind rhythmic output; (3) expression of these genes is rhythmic and inhibited either directly or indirectly by the translation products. $(4,5)$ In Drosophila, PER and TIM form a complex, $(30,31)$ move to the nucleus and down-regulate the CYC-dCLOCK complex that activates per and tim expression.(32-34) In Neurospora, expression of frq is repressed by FRQ and the WC1:WC2 protein complex appears to function as an activator of frq's autoregulatory oscillation. A similar model has been proposed in mice, involving mPER and BMAL1 and CLOCK. ${ }^{(35)}$ (See Ref. 5 for a review of eukaryotic clock models.)

How do the kai genes generate the rhythm in cyanobacteria? Transcriptional analysis should provide important information to address this question. (27) The kai gene cluster of Synechococcus is transcribed by two promoters, PkaiA (for kaiA) and PkaiBC (for kaiB and kaiC). Both promoters mediate rhythmicity (of the same phase) for the respective mRNAs. Overexpression of the kaiC gene immediately and completely represses its own PkaiBC promoter. This strong negative autoregulation of kaiC expression by KaiC suggests that KaiC functions as the key component of the circadian oscillation of cyanobacteria (as the oscillating object in the physical model does). On the other hand, inactivation of kaiA lowers PkaiBC activity and kaiA overexpression dramatically increases PkaiBC activity. As expression of kai $A$ is in phase with kaiBC activity, kaiBC expression seems to positively autoregulated by KaiA. Thus, KaiA might function as a positive factor that generates or sustains the KaiC oscillation. Figure 1 illustrates the model of Synechococcus oscillator based on expression of kai genes and Kai proteins.
If expression of clock genes and the proteins they encode are key components of the circadian oscillator, such that the level of the expression directly defines the phase of the oscillation, a temporal alteration in the expression should alter the phase of the oscillation. Alternatively, light pulses that shift the phase of the rhythm should alter the expression of these genes. In Drosophila, light pulses promote degradation of the PER/TIM complex(36,37) and alteration in the expression of frq in Neurospora and mper in mice by a light pulse explains light-induced phase shifts of their biological rhythms. ${ }^{(38,39)}$ In Synechococcus, over-expression of kaiC, shifts the phase of the oscillation in a way predicted by the kai-based model.(27) The phase was advanced by overexpression of kaiBC when PkaiBC activity was on the rise and delayed when PkaiBC product levels waned. Thus, these results favor a model in which expression of the kaiC gene and its products function as the key components of the Synechococcus oscillator.

\section{Cycling by the circadian period}

It is possible that the clock genes identified to date do not form the central circadian oscillator. An alternate possibility, that the loops so far identified function in closely related peripherals of the oscillator, is still possible.(40) Many questions about the circadian mechanism remain to be answered. In the case of Synechococcus, transcription factors that form the feedback loop need to be identified, for no DNA-binding motif has been identified in the Kai proteins. ${ }^{(27)}$

At present, no clock model explains the molecular mechanism that accounts for the key feature of the circadian oscillations, the length and stability of the $24 \mathrm{~h}$ rhythm (see below). As mentioned previously, single amino acid substitutions in KaiC alter the period of the Synechococcus rhythm to as short as $14 \mathrm{~h}$ or as long as $60 \mathrm{~h}$.(27) At present, no biochemical function of the Kai protein can be predicted from the amino acid sequence, except for two ATP/GTP binding domains (Walker's p-loop) have been identified in KaiC. These domains seem to be important for the generation of circadian oscillation, because mutations in those motifs greatly alter rhythmicity (unpublished data).

In eukaryotic clock systems, protein-protein complexes (mediated via PAS domains) are thought to be crucial for generating the circadian oscillation. In Drosophila, cytoplasmic accumulation of PER and TIM and phosphorylation of PER(41) leads to formation of a heterodimer that can move to the nucleus, where it is thought to reduce per and tim transcription by inhibiting the CLK-dCLOCK activity. $(4,5,42)$ In Synechococcus, various heterotypic and homotypic associations among the three Kai proteins have been demonstrated in yeast cells (using the two-hybrid assay system), in vitro (by the GTS-pull-down assay), and in Synechococcus cells. Moreover, a long period mutation, kaiA1, enhanced these interactions. ${ }^{(43)}$ Homodimeric interaction of KaiB in 
vivo has also been demonstrated.(44) These results suggest that physical interactions among the Kai proteins are important for generation of circadian rhythms in Synechococcus.

Time delay in the feedback process is expected to be important in generating the period of circadian oscillators. The clock models of Drosophila and Neurospora assume that an important timing mechanism of a circadian feedback loop is the transport of the clock proteins (PER, TIM, FRQ and $\mathrm{mPER}$ ) from the cytoplasm, where they are synthesized, to the nucleus, where they affect the expression of the genes encoding these proteins. The time required for the nuclear translocation of the clock protein(s) is postulated to account for a period as long as 24 h. $(4,5,42)$ Thus, in eukaryotic organisms, cell division, which disrupts and divides the nuclear structure, might interfere with this feature of circadian timing mechanisms. In fact, no circadian rhythm has been reported for cells dividing faster than the circadian period.(3) Cyanobacteria have no nuclear envelope, however. Moreover, Synechococcus that divide as rapidly as $6 \mathrm{~h}$ show robust circadian rhythmicity. This was demonstrated in continuously diluted cultures ${ }^{(45)}$ and confirmed by curve fitting of observed bioluminescence to an exponential growth model of nondiluting culture.(46) Thus, if a similar mechanism applies in prokaryotes, cell division and DNA replication events must either be less disruptive to the circadian functions than in eukaryotes, or an alternative compartment must serve the sequestration function in cyanobacteria. It is possible that the eukaryotic clock has evolved to function in eukaryotic genetic system, and intraorganelle events may have been incorporated that have no parallel in cyanobacteria.

\section{Evolution of the circadian clock}

Similarities between physiological characteristics of circadian rhythms among diverse organisms anticipate that the unique daily clock system evolved only once, and has been evolutionarily conserved, as is the case for many fundamental biochemical processes. The proteins encoded by clock genes in different organisms do not share sequence similarity, however. (5) It appears more likely that the clock systems developed independently in cyanobacteria, fungi, and animals, and probably also in plants. Natural selection by the alternating environment apparently creates pressure to promote the evolution of similar clock properties. This idea should not be surprising because negative feedback of gene expression is the rule in living cells, rather than the exception. In each group of organisms, one (or a few) feedback systems has developed mechanisms for self-sustained oscillation (necessarily associated with activator components) and temperature-compensation of periods, each evolving analogous $24 \mathrm{~h}$ clocks.

To explore these evolutionary issues within bacteria, our search for homologues of the kai gene cluster has identified homologues in various species of cyanobacteria such as
Anabaena sp. PCC 7120, Synechococcus vulcanus, and Synechocystis sp. PCC 6803. Thus, kai genes are common to the cyanobacteria group, while homologues of kai genes are not found in databases of eubacteria, and no circadian rhythm has been found in prokaryotes other than cyanobacteria. Putative KaiB and KaiC homologues are found in archaeal genome, however. While no physiological function for these proteins in Archaea has been identified, a phylogenetic tree based on these sequences suggests that cyanobacterial KaiC is derived from Archaea (Ishiura et al., in preparation).

Another interesting point is the possible relationship between the clock of cyanobacteria and that of higher plants. Is the cyanobacteria clock the ancestor of plant clocks, as is the case for the factors subserving photosynthesis? It is conceivable that kai genes were incorporated into ancestral plant cells along with photosynthetic genes. While molecular cycling models have not yet proposed for plants, several studies have identified clock-related genes.(47-49) But, no homologue of Kai has been found in the plant genomes so far sequenced. Thus, it may be that plants evolved a clock system independently based on an entirely different origin. Even if the proposed evolutionary transfer occurred, an oscillator based on Kai must have changed considerably, since prokaryotic gene regulation would be subject to modification after translocation to the eukaryotic nucleus.

\section{Future directions}

Molecular genetic dissection of the circadian system of cyanobacteria has revealed the basic structure of Kai-based oscillator. As cyanobacteria are amenable to molecular analysis, further studies might make the current model more realistic by uncovering as yet unknown links in the oscillatory loop and biochemical mechanisms that account for circadian period and its stability. Moreover, understanding the cyanobacterial oscillator will shed light on the phylogeny of the circadian system in diverse organisms.

\section{Acknowledgments}

We thank Drs. C. H. Johnson (Vanderbilt University) and S. S. Golden (Texas A\&M University) for fruitful collaboration on the cyanobacterial clock.

\section{References}

1. Bünning E. The physiological clock, $3^{\text {rd }}$ ed. New York: Springer Verlag; 1973.

2. Pittendrigh CS. Temporal organization: reflections of a Darwinian clockwatcher. Annu Rev Physiol 1993; 55:17-54.

3. Edmunds LN. Cellular and molecular bases of biological clocks. New York: Springer Verlag; 1988.

4. Wilsbacher LD, Takahashi JS. Circadian rhythms: molecular basis of the clock. Curr Opin Genet Develop 1998;5:595-602

5. Dunlap J. Molecular basis for circadian clocks. Cell 1999;96:271-290.

6. Golden SS, Ishiura M, Johnson $\mathrm{CH}$ Kondo T. Cyanobacterial circadian rhythms. Annu Rev Plant Physiol Plant Mol Biol 1997;48:327-354. 
7. Mitsui A, Kumazawa S, Takahashi A, Ikemoto H, Cao S, Arai T. Strategy by which nitrogen-fixing unicellular cyanobacteria grow photoautotrophically. Nature 1986;323:720-722.

8. Huang T-C, Tu J, Chow T-J, Chen T-H. Circadian rhythm of the prokaryote Synechococcus sp. RF-1. Plant Physiol 1990;92:531-533.

9. Chen T-H, Chen T-L, Hung L-M, Huang T-C. Circadian rhythm in amino acid uptake by Synechococcus RF-1. Plant Physiol 1991;97:55-59.

10. Huang T-C, Chen H-M, Pen S-Y, Chen T-H. Biological clock in the prokaryote Synechococcus RF-1. Planta 1994;193:131-136.

11. Sweeney BM, Borgese MB. A circadian rhythm in cell division in a prokaryote, the cyanobacterium Synechococcus WH7803. J Phycol 1989;25:183-186. 12. Schneegurt MA, Sherman DM, Nayar S, Sherman LA. Oscillating behavior of carbohydrate granule formation and dinitrogen fixation in the cyanobacterium Cyanothece sp. strain ATCC 51142. J Bacteriol 1994;176:15861597.

13. Roenneberg $\mathrm{T}$, Carpenter EJ. Daily rhythm of $\mathrm{O}_{2}$-evolution in the cyanobacterium Trichodesmium thiebautii under natural and constant conditions. Marine Biol 1993;117:693-697.

14. Morse DS, Fritz L, Hastings JW What is the clock? Translational regulation of circadian bioluminescence. Trends Biochem Sci 1990;15:262-265.

15. Somers DE, Webb AA, Pearson M, Kay SA. The short-period mutant, toc1-1, alters circadian clock regulation of multiple outputs throughout development in Arabidopsis thaliana. Development 1998;125:485-494.

16. Stanewsky R, Kaneko M, Emery P, Beretta B, Wager-Smith K, Kay SA, Rosbash M, Hall JC. The cryb mutation identifies cryptochrome as a circadian photoreceptor in Drosophila. Cell 1998;95:681-692.

17. Tsinoremas NF, Kutach AK, Strayer CA, Golden SS. Efficient gene transfer in Synechococcus sp. strain PCC 7942 and PCC 6301 by interspecies conjugation and chromosomal recombination. J Bacteriol 1994;176:6764-678.

18. Golden SS. Light-responsive gene expression and the biochemistry of the photosystem II reaction center. In: Bryant DA, editor. The molecular biology of cyanobacteria. Dordrecht, The Netherlands: Kluwer Academic; 1994. p 693714.

19. Kondo T, Strayer CA, Kulkarni RD, Taylor W, Ishiura M, Golden SS, Johnson $\mathrm{CH}$. Circadian rhythms in prokaryotes: luciferase as a reporter of circadian gene expression in cyanobacteria. Proc Natl Acad Sci USA 1993;90:56725676 .

20. Liu Y, Tsinoremas NF, Johnson CH, Lebedeva NV, Golden SS, Ishiura M, Kondo T. Circadian orchestration of gene expression in cyanobacteria. Genes Develop 1995; 9:1469-1478.

21. Tsinoremas NF, Ishiura M, Kondo T, Andersson CR, Tanaka K, Takahashi H, Johnson $\mathrm{CH}$, Golden SS. A sigma factor that modifies the circadian expression of a subset of genes in cyanobacteria. EMBO J 1996;15:2488-2495.

22. Katayama M, Tsinoremas NF, Kondo T, Golden SS. cpmA, a gene involved in an output pathway of the cyanobacterial circadian system. J Bacteriol 1999; 181:3516-3524

23. Liu Y, Tsinoremas NF, Golden SS, Kondo T, Johnson CH. Circadian expression of genes involved in the purine biosynthetic pathway of the cyanobacterium Synechococcus sp. strain PCC 7942. Mol Microbiol 1996;20:10711081.

24. Johnson CH, Golden SS, Kondo T. Adaptive significance of circadian programs in cyanobacteria. Trends Microbiol 1998;6:407-410.

25. Klarsfeld A, Rouyer F. Effects of circadian mutations and LD periodicity on the life span of Drosophila melanogaster. J Biol Rhythms 1998;13:471-478.

26. Kondo T, Tsinoremas NF, Golden SS, Johnson CH, Kutsuna S, Ishiura M. Circadian clock mutants of cyanobacteria. Science 1994;266:1233-1236.

27. Ishiura M, Kutsuna K, Aoki S, Iwasaki H, Andersson CR, Tanabe A, Golden SS, Johnson $\mathrm{CH}$, Kondo T. Expression of a gene cluster kaiABC as a circadian feedback process in cyanobacteria. Science 1998;281:1519-1523.

28. Kutsuna S, Kondo T, Aoki S, Ishiura M. A period-extender gene, pex, that extends the period of the circadian clock in the cyanobacterium Synechococcus sp. PCC 7942. J Bacteriol 1998;180:2167-2174.
29. Goldbeter A. Biochemical oscillations and cellular rhythms. Cambridge: Cambridge University Press; 1996.

30. Saez L, Young MW. Regulation of nuclear entry of the Drosophila clock proteins Period and Timeless. Neuron 1996;17:911-920.

31. Zeng H, Hardin PE, Rosbash M. Constitutive overexpression of the Drosophila period protein inhibits period mRNA cycling. EMBO J 1994;13:35903598.

32. Allada R, White NE, So WV, Hall JC, Rosbash M. A mutant Drosophila homolog of mammalian Clock disrupt circadian rhythms and transcription of period and timeless. Cell 1998;93:791-804.

33. Rutila JE, Suri V, Le M, So WV, Rosbash M, Hall JC. CYCLE is a second bHLH-PAS clock protein in essential for circadian rhythmicity and transcription of Drosophila period and timeless. Cell 1998;93:805-814.

34. Darlington TK, Wager-Smith K, Ceriani MF, Staknis D, Gekakis N, Steeves DL, Weitz CJ, Takahashi JS, Kay SA. Closing the circadian feedback loop: CLOCK induced transcription of its own inhibitors, period and timeless. Science 1998;280:1599-1603.

35. Gekakis N, Staknis D, Nguyen HB, Davis FC, Wilsbacher LD, King DP, Takahashi JS, Weitz CJ. Role of the CLOCK protein in the mammalian circadian mechanism. Science 1998;280:1564-1569.

36. Zeng H, Qian Z, Myers MP, Rosbash M. A light-entrainment mechanism for the Drosophila circadian clock. Nature 1996;380:129-135.

37. Hunter-Ensor M, Ousley A, Sehgal A. Regulation of the Drosophila protein Timeless suggests a mechanism for resetting the circadian clock by light. Cell 1996;84:677-685.

38. Crosthwaite SK, Loros JJ, Dunlap JC. Light-resetting of a circadian clock is mediated by a rapid increase in frequency transcript. Cell 1996;81:10031012.

39. Shigeyoshi Y, Taguchi K, Yamammoto S, Takehida S, Yan L, Tei H, Moriya T, Shibata S, Loros JJ, Dunlap JC, Okamura H. Light-induced resetting of a mammalian circadian clock is associated with rapid induction of the $\mathrm{mPer} 1$ transcript. Cell 1997,91:1043-1053.

40. Merrow M, Brunner M, Roenneberg T. Assignment of circadian function for the Neurospora clock gene frequency. Nature (London) 1999;399:584-586.

41. Kloss B, Price JL, Saez L, Blau J, Rothenfluh A, Wesley CS, Young MW. The Drosophila clock gene double-time encodes a protein closely related to human casein kinase le. Cell 1998;94:97-107.

42. Young MW. The molecular control of circadian behavioral rhythms and their entrainment in Drosophila. Annu Rev Biochem 1998;67:135-152.

43. Iwasaki $H$, Taniguchi $Y$, Ishiura $M$, Kondo $T$. Physical interactions among circadian clock proteins, KaiA, KaiB and KaiC, in cyanobacteria. EMBO J 1999;18:1137-1145.

44. Xu Y, Piston DW, Johnson $\mathrm{CH}$. A bioluminescence resonance energy transfer (BRET) system: Application to interacting circadian clock proteins. Proc. Natl Acad Sci USA 1999;96:151-156.

45. Mori T, Binder B, Johnson $\mathrm{CH}$. Circadian gating of cell division in cyanobacteria growing with average doubling times of less than 24 hours. Proc Natl Acad Sci USA 1996;93:10183-18186.

46. Kondo T, Mori T, Lebedeva NV, Aoki S, Ishiura M, Golden SS. Circadian rhythms in rapidly dividing cyanobacteria. Science 1997;275:224-227.

47. Millar AJ, Carré IA, Strayer CA, Chua N-H, Kay SA. Circadian clock mutants in Arabidopsis identified by Luciferase imaging. Science 1995;267:11611166.

48. Wang ZY, Tobin EM. Constitutive expression of the CIRCADIAN CLOCK ASSOCIATED 1 (CCA1) gene disrupts circadian rhythms and suppresses its own expression. Cell 1998;93:1207-1217.

49. Schaffer R, Ramsay N, Samach A, Corden S, Putterill J, Carre IA, Coupland G. The late elongated hypocotyl mutation of Arabidopsis disrupts circadian rhythms and the photoperiodic control of flowering. Cell 1998;93: 1219-1229. 